\title{
El trasplante en Navarra y España
}

\section{Transplantation in Navarra and Spain}

\author{
J. J. Unzué
}

\section{RESUMEN}

La donación de órganos y tejidos es un proceso admitido en nuestra sociedad como algo habitual, así ante la inminencia de la muerte, y el planteamiento de la gravedad del paciente, en una UCI, la familia ofrece los órganos y/o tejidos antes de solicitarlos. Como cifra clave, al finalizar el año 2005, se produjeron tres datos históricos: por un lado, en España ha pasado de 35 donaciones por millón -jrécord mundial!-, y por otro lado, se ha conseguido la cifra más baja de negativas familiares de la historia, situándonos por debajo del 16 por millón, y además se ha podido rebasar la cifra de 1.500 donantes en un año.

El hecho de llevar más de 30 años de experiencia en donación y trasplante ha permitido optimizar métodos y protocolos y distribuir con eficacia funciones sobre la base de la especialización; el Hospital de Navarra se encarga preferentemente de la extracción de órganos; el Hospital Virgen del Camino se ocupa de la captación de tejidos, y la Clínica Universitaria de Navarra del proceso quirúrgico del trasplante.

En este trabajo se ofrecen datos de los distintos trasplantes haciendo referencia continuamente a los datos producidos entre los años 1995 y 2005; cuando la ocasión lo requiere, se citan también datos anteriores y se establecen algunas comparaciones con el resto de España y otros países.

Palabras clave. Donación de órganos y tejidos. Generosidad. Negativas familiares.

An. Sist. Sanit. Navar. 2006; 29 (Supl. 2): 7-14.

\begin{abstract}
The donation of organs and tissues is accepted in our society as a normal process; thus it frequently happens that when, in the face of imminent death, we inform the family of the severity of the patient in an intensive care unit, they offer us the organs and/or tissues before they are even asked. That is to say that in our milieu the degree of awareness and generosity is very high. As a sort of magic figure, three historic events occurred at the end of 2005: on the one hand, Spain exceeded the figure of 35 donations per million a world record; on the other hand, we obtained the lowest figure of family refusals in history, that is, we are now below 16 per million, which is also a magic figure that is unique in the world; and, besides, we exceeded the figure of 1,500 donations in one year.

The experience of 30 years of donations and transplantations has enabled us to optimise methods and protocols and to efficiently distribute functions on the basis of specialisation. The Hospital of Navarra preferentially takes charge of the extraction of organs; the Virgen del Camino Hospital collects tissues; and the University Clinic of Navarra carries out the surgical process of the transplantion.

In this article we present data on the different transplants, making continuous reference to the figures produced between the years 1995 and 2005; we refer to previous figures and we make a comparison with the rest of Spain and the world.
\end{abstract}

Key words. Organ and tissue donation. Generosity. Family refusals.
Coordinador Autonómico de Trasplantes y Tejidos

\author{
Correspondencia: \\ Juan José Unzué Gaztelu \\ Coordinador Autonómico de Trasplantes y \\ Tejidos \\ Departamento de Salud \\ Amaya 2-A, $3^{\text {o }}$ \\ 31002 Pamplona. Navarra \\ Teléfono 848423532 \\ Fax 848421444 \\ E-mail junzuega@cfnavarra.es
}




\section{TRASPLANTE RENAL}

Es el trasplante más numeroso en nuestra comunidad, al igual que en todo el mundo (Fig. 1).

En Navarra, se implantó la técnica del trasplante renal en la Clínica Universitaria de Navarra en el año 1969. La técnica se ha ido consolidando poco a poco ya que en 1969, se trasplantó un solo paciente y en 1970, ninguno; en 1971 fueron 2 y el ritmo fue creciendo paulatinamente en los años ochenta, noventa y dos mil, habiendo realizado 709 trasplantes en estos 25 años $^{1}$. La evolución del trasplan- te en España puede observarse en la figura 2.

Los pacientes que sufren el trasplante provienen de la diálisis, ya sea de la hemodiálisis (HD) o de la diálisis peritoneal (DP); en HD el paciente normalmente acude a diálisis tres veces por semana, entre tres y cinco horas cada sesión. Paralelamente a esta técnica va desarrollándose con ritmo creciente la hemodiálisis domiciliaria que ofrece evidentes ventajas: el paciente se puede dializar en su propio domicilio evitando la aversión y desarraigo que en ocasiones producen

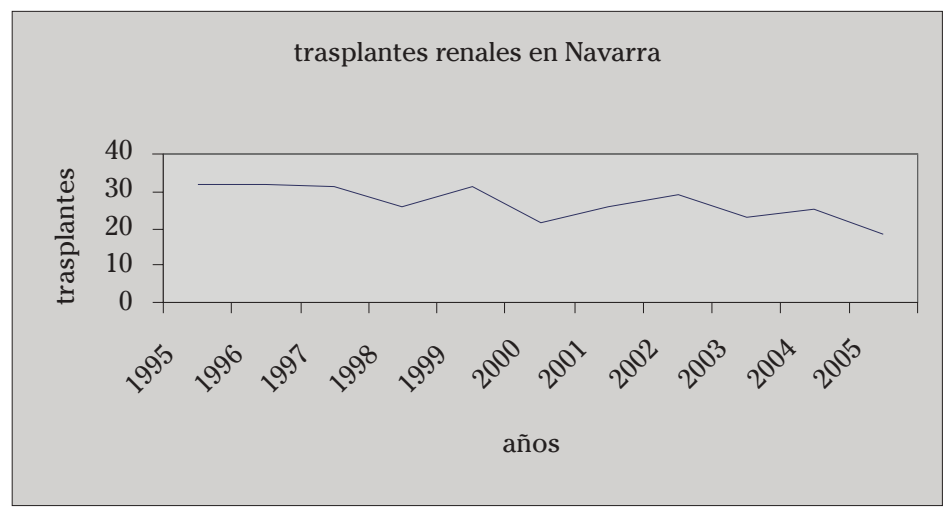

Figura 1. Trasplante renal en Navarra de los años 1995 al 2005.

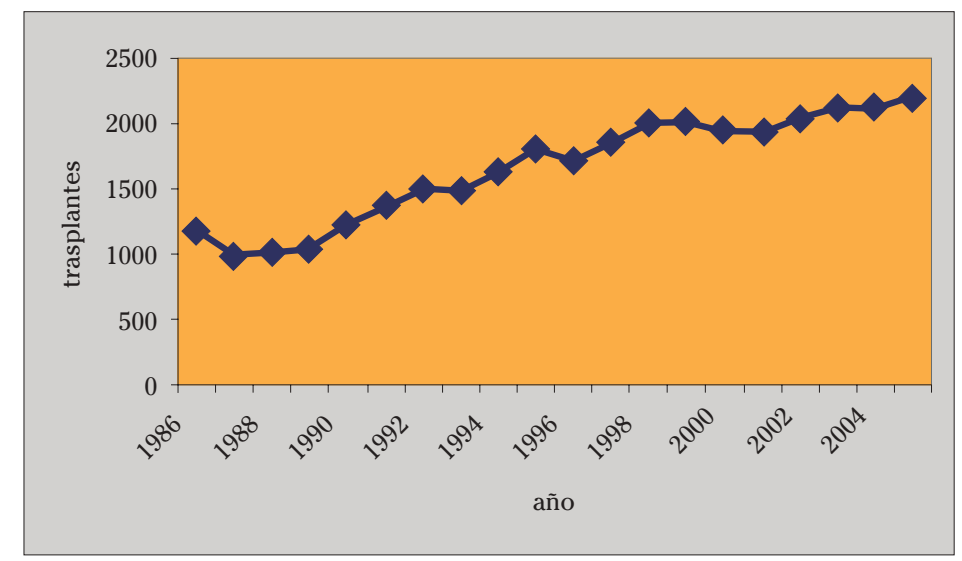

Figura 2. Trasplante renal en España de los años 1995 al 2005. 
los centros hospitalarios, la incomodidad del transporte y la posibilidad de escoger horarios y situaciones. La DP se realiza generalmente en el domicilio del paciente diariamente y existen varias modalidades: una manual, que consiste en la introducción de una solución en la cavidad peritoneal que permanecerá durante un intervalo de tiempo y se extraerá posteriormente. Esta operación se repite entre tres y cinco veces al día o bien, automáticamente introduciendo el líquido peritoneal y extrayéndolo durante toda la noche en una serie de ciclos hasta unos diez o doce en cada noche. Esta modalidad permite al paciente disponer del día para realizar actividades de todo tipo, olvidándose de la diálisis. En este caso la membrana de diálisis es el peritoneo y esta modalidad se llama diálisis peritoneal continua (DPCA) y la nocturna, que es automática, es la denominada diálisis peritoneal automática (APD).

El número de pacientes en diálisis en todo el mundo está aumentado cada año y las cifras son preocupantes, en gran parte por dos lacras de la sociedad actual desarrollada, como son la diabetes y la hipertensión arterial; así, en Navarra el número de pacientes en espera de un trasplante renal a finales del año 2005 era de 58.

En España el número de pacientes en diálisis está aumentado, y paralelamente la lista de espera también, así a final del 2005 había en España 4.200 personas a la espera de un trasplante renal' ${ }^{2}$.

En el resto del mundo el número de trasplantes renales depende de un conjunto de variables como son: el nivel económico, la situación geográfica, la conciencia social y otros factores diversos en cada país $^{36}$.

Un trasplante que está aumentando día a día en nuestros hospitales es el trasplante de riñón-páncreas conjuntamente ${ }^{7}$. La complejidad de la técnica hacía la operación muy difícil y con resultados muy inciertos, pero actualmente se realiza en nuestros hospitales españoles con resultados muy alentadores. Con este trasplante se consigue que el paciente diabético en diálisis, reciba en el mismo acto quirúrgico ambos órganos y con ello se evita la diálisis y el tratamiento con insulina. En nuestra comunidad no tenemos instaurada la técnica para realizar este doble trasplante y por ello, los escasos pacientes que podrían ser candidatos a la misma, unos tres por año, son remitidos al Hospital Clínic de Barcelona.

La verdad es que lograr que un paciente diabético sometido a diálisis, se libere de la diálisis y la insulina, suena más a ciencia-ficción que a hipótesis realistas. Los pacientes con esta doble dolencia y trasplantados con éxito no encuentran palabras para describir sus vivencias y tienen la sensación de estar viviendo una nueva vida.

Otra modalidad de trasplante renal, es el trasplante renal de donante vivo, es decir la recepción del riñón de un familiar sano y, por tanto, quedando el donante con un solo riñón. Así en España, el año 2005, se hicieron 85 trasplantes de este tipo y el año 1991 fueron 16. Este tipo de trasplante supone el 3,8\% de los realizados en España ${ }^{8}$. En Navarra es anecdótico ya que sólo se realizan uno o dos al año.

Otra indicación de trasplante es la llamada prediálisis. La prediálisis es un estado de desarrollo de una enfermedad, merced a la cual y en un corto período de tiempo el paciente será incluido en diálisis, pero es en ese período de prediálisis, si surge la ocasión favorable, puede ser trasplantado sin pasar por la diálisis; son pocos los que disfrutan de esta técnica. En Navarra tan solo son uno o dos al año.

\section{TRASPLANTE HEPÁTICO}

Hace años cuando se producía un trasplante hepático, cardíaco o renal, era portada de la prensa local, hoy en día esto ya no es noticia, ya que es práctica habitual en los hospitales españoles. Esta modalidad terapéutica ha ido aumentando los 20 últimos años en España y así, en el año 1984, se trasplantaron en este país 14 pacientes y en el año 2005, 1.070 pacientes (Fig.3) $^{9-11}$.

En la comunidad de Navarra, los datos desde 1995 hasta 2005 se recogen en la figura 4 . 
Al día de hoy, en Navarra, la lista de espera es de 6 pacientes; en el resto de España es de 608. En otros países las cifras son muy variables. El número de trasplantes hepáticos en España supera al de cualquier otro país.

\section{TRASPLANTE CARDÍACO}

Este trasplante es habitual en los hospitales españoles. Así, el año 2005, se trasplantaron en nuestro país 287 corazones y la lista de espera fue de 85 (Fig. 6). La nece-

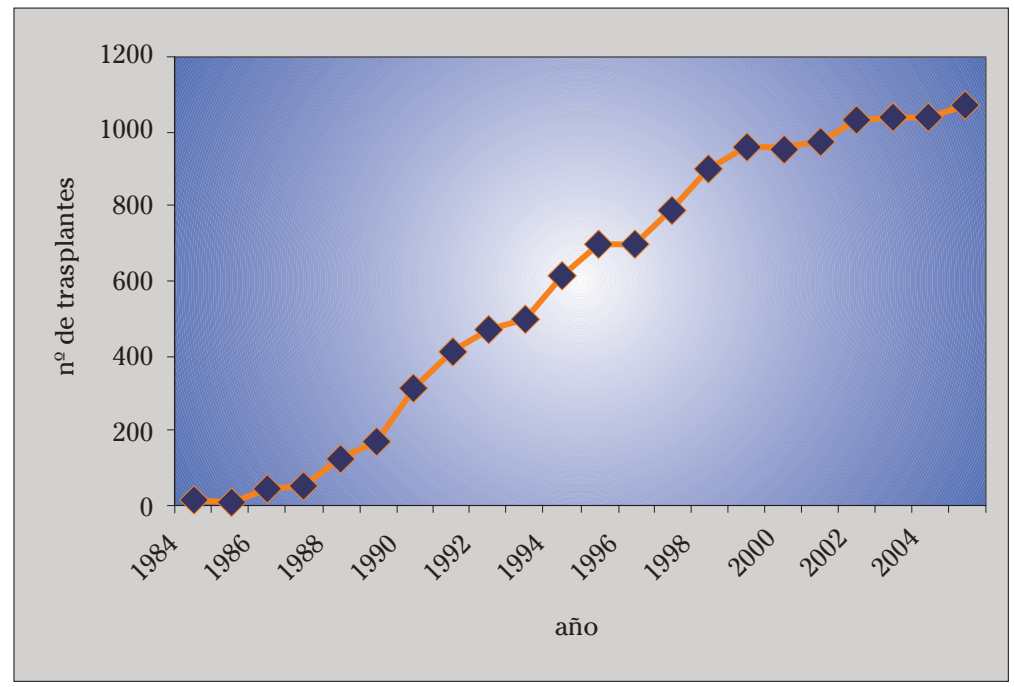

Figura 3. Trasplante hepático en España.

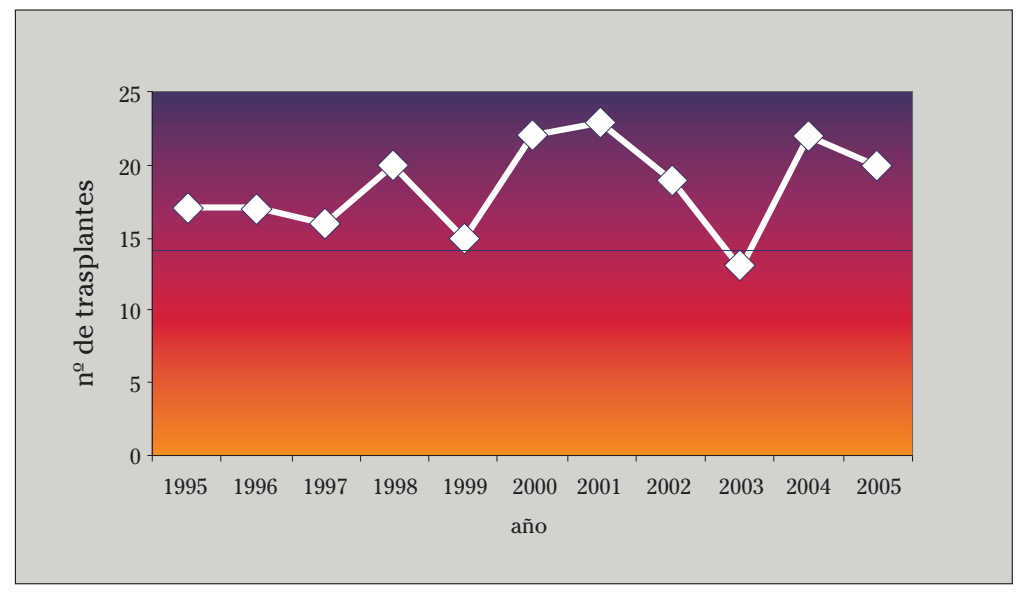

Figura 4. Trasplante hepático en Navarra. 
sidad de trasplantes cardíacos ha descendido en todo el mundo gracias a la medicación y a las nuevas tecnologías: by-pass, stent, etc.; en definitiva, ha habido un considerable desarrollo de las nuevas tecnologías cardíacas que han hecho menos necesaria esta modalidad de trasplante. La evolución del trasplante cardiaco en Navarra se refleja en la figura 5 .

En el año 1984 se trasplantaron en España 10 corazones y en el año 2005, 287, pero hace ocho años se hicieron 349 trasplantes de corazón por lo que podemos comprobar que las necesidades son menores que hace sólo unos pocos años ${ }^{12,13}$. En el mundo, estamos a la cabeza en donaciones y trasplantes.

\section{TRASPLANTE PULMONAR}

El trasplante de pulmón, al igual que el de otros órganos, en España, ha crecido en los últimos años. Así, si comparamos los

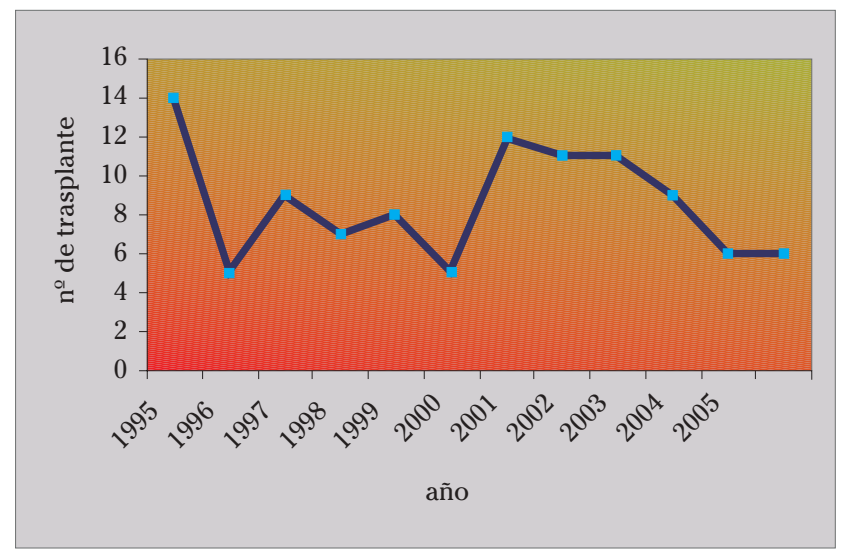

Figura 5. Trasplante cardíaco en Navarra.

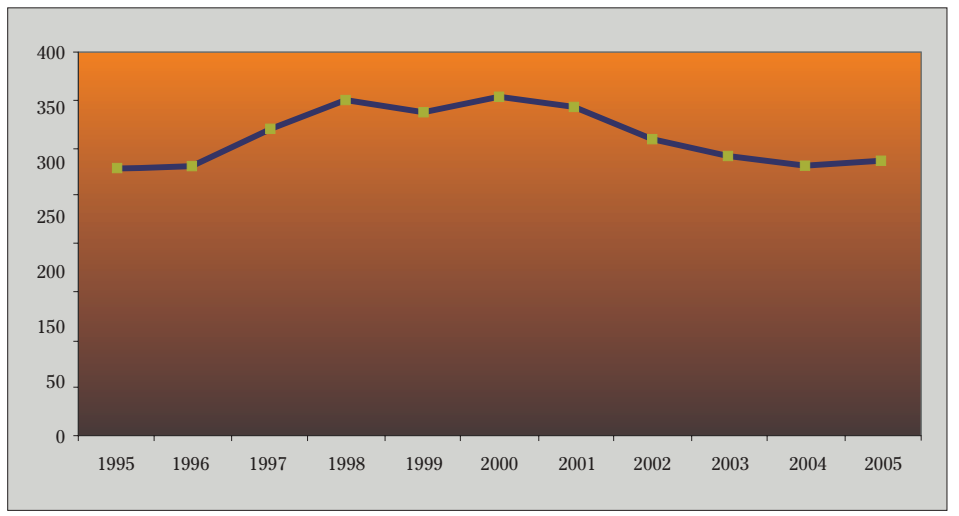

Figura 6. Trasplante cardíaco en España. 
últimos años, se puede observar que desde 1990 hasta 2005 las cifras han sido: $6,6,11,20 \ldots$ hasta 2005 , que fueron de 167 (Fig. 7).

El conseguir donantes pulmonares no es tarea fácil, ya que una vez detectado el posible donante entran en juego una serie de factores tales como la hipertensión, tiempo de estancia en la UCI, tiempo de intubación, tratamiento con antibióticos, presión parcial de oxígeno, que pueden hacer inviable el trasplante; en definitiva, que de 100 donantes solamente lo son de pulmón 10 es decir el 10\%. La lista de espera de trasplante de pulmón en España, a 31 de diciembre de 2005, era de 127 y en 2004 , era de $145^{14,15}$.

En nuestra comunidad el número de pacientes en lista de espera es exiguo y los pacientes son referidos al Hospital de Valdecilla (Cantabria), ya que Navarra carece del equipo adecuado para efectuar este trasplante.

\section{OTROS TRASPLANTES}

Existen otros trasplantes mucho menos frecuentes en todo el mundo como el trasplante de intestino que se inició en 1999 y el número de trasplantes realizados es de 3 ó 4 al año en centros muy señalados de España, como la el Hospital Universitario La Paz, el Hospital Ramón y Cajal y 12 de Octubre.
Otros trasplantes combinados son: corazón-hígado, hígado-intestino, corazónpulmón, hígado-páncreas, hígado-pulmón, páncreas-riñón, etc. son poco frecuentes y se realizan esporádicamente en diversos hospitales españoles ${ }^{16}$.

\section{TRASPLANTE DE TEJIDOS}

Cada vez son más los trasplantes de tejidos que se realizan en todo el mundo. Navarra cuenta con un programa específico de trasplantes de tejidos dentro del programa "Pamplona". Precisamente gracias a esos donantes se mantiene prácticamente a cero la lista de espera de tejidos, tales como córneas, sangre, médula ósea, huesos o válvulas entre otros ${ }^{17}$.

\section{Sangre y médula ósea}

Los trasplantes de progenitores hematopoyéticos (THP) constituyen hoy día una terapéutica establecida para gran variedad de enfermedades congénitas y adquiridas que afectan a la médula ósea. Los tradicionales trasplantes de médula ósea procedentes de un hermano HLA idéntico están siendo sustituidos por los de células progenitoras hematopoyéticas procedentes de otros orígenes como la sangre periférica o la sangre de cordón umbilical, así como la procedente de otro tipo de donantes como son otros familiares y donantes no emparentados. Actualmente existen en

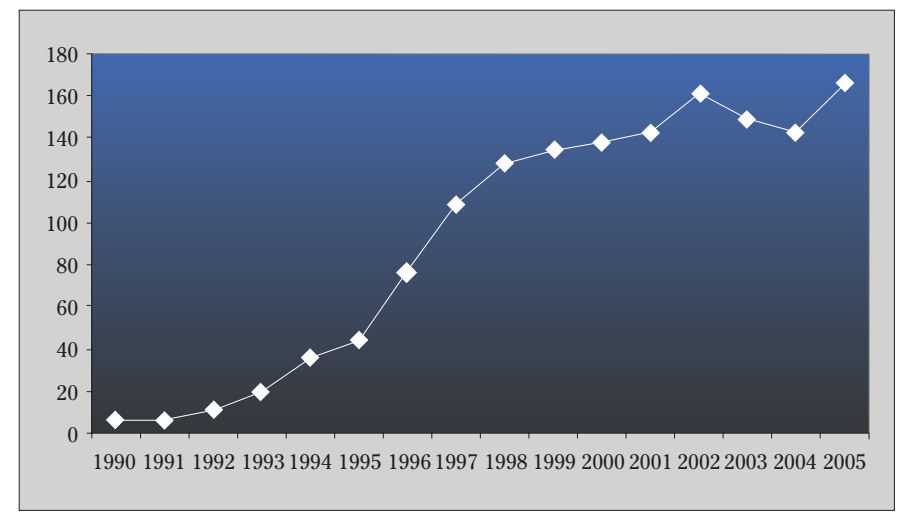

Figura 7. Trasplante pulmonar en España. 
el mundo cerca de 10 millones de donantes de médula ósea y más de 150.000 de sangre de cordón umbilical (SCU) lo que evidentemente ha incrementado la posibilidad de encontrar un donante compatible y la posibilidad de realización de un trasplante no emparentado ${ }^{18}$.

En los últimos cuatro años ha habido un incremento del número de trasplantes de progenitores hematopoyéticos realizados en nuestro país y concretamente por autonomías la primera ha sido Cantabria, seguida de Navarra y Cataluña ${ }^{19}$.

\section{Trasplante de córnea}

El trasplante de tejido corneal, lo que nosotros llamamos córneas, es francamente alentador y ha aumentado mucho la donación de córneas en los últimos años. Así en el año 2005 se obtuvieron en España 5.759 córneas efectuándose 2.758 trasplantes ${ }^{20}$. En Navarra se obtuvieron 96 y se hicieron 33 trasplantes ${ }^{21}$ (Fig. 8).

\section{Trasplante de músculo-esquelético}

Un trasplante no suficientemente valorado es el de músculo-esquelético; es muy frecuente. Navarra está a un buen nivel nacional y autonómico y, si no es objeto de grandes comentarios es porque no tiene relevancia mediática. Resulta eficaz en muchos casos y resuelve muchos problemas menores ${ }^{22}$.

\section{Membrana amniótica}

Un trasplante de plena actualidad es el de membrana amniótica. La técnica es muy reciente. Los datos son muy alentadores y cada vez se aplica más ${ }^{23}$.

\section{Cultivos celulares}

El cultivo celular es una práctica incipiente, implantada en algunos hospitales españoles y creemos que estamos ante un reto de futuro que podría desarrollarse considerablemente, en consonancia con la terapia génica ${ }^{24}$.

\section{Válvulas cardíacas}

Un tema que está aumentando en todo el mundo es el trasplante de válvulas cardíacas y segmentos vasculares ${ }^{25,26}$. En Navarra se montó esta técnica en 2004 con buenos resultados; las cifras son poco significativas porque Navarra es una comunidad pequeña y la muestra es de escasa relevancia; el peso específico lo llevan las comunidades más grandes: Andalucía, Cataluña, Madrid, Galicia y Valencia.

\section{CONCLUSIÓN}

España se encuentra a la cabeza del mundo en donación-trasplante. Esta labor es el día a día de un equipo de personas anónimas que trabajan en silencio, tanto profesionales sanitarios como personas

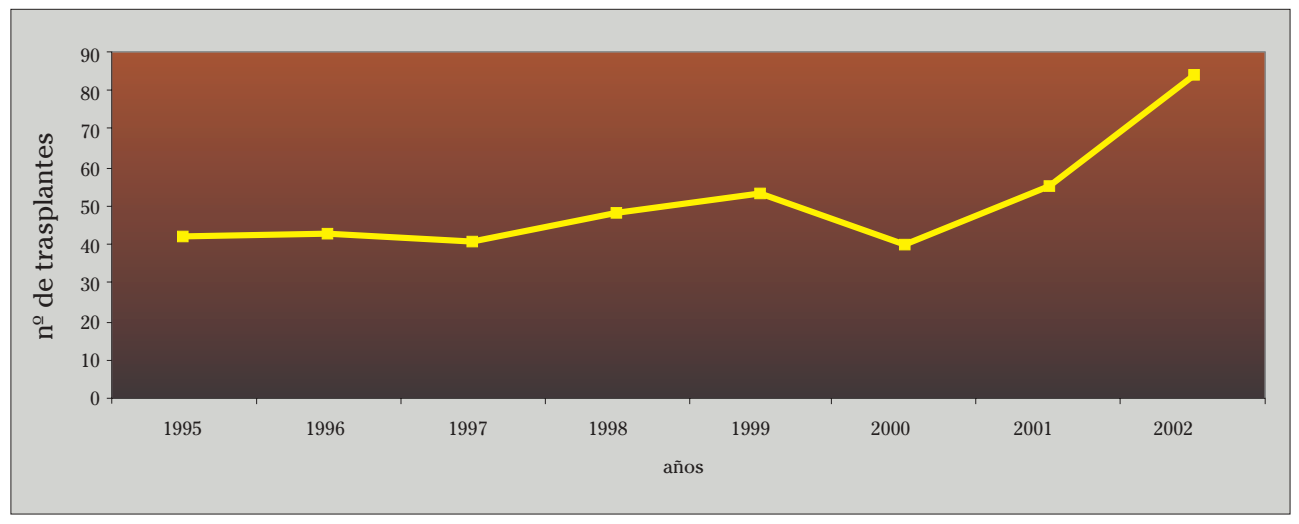

Figura 8. Trasplante de córnea en Navarra. 
que intervienen en el proceso del trasplante y que hacen que se extraigan los órganos y tejidos en condiciones favorables para poder trasplantar, porque efectivamente, si no hay donantes no hay trasplantes. Todo el personal es necesario en la ejecución de sus tareas específicas y todos contribuyen a consolidar la cadena con la conciencia clara de que la ruptura de un solo eslabón haría inviable todo el proceso.

Tenemos a día de hoy un programa de donación - trasplante en nuestra Comunidad, que en palabras oídas en otras autonomías y países, es un modelo a exportar a cualquier parte del mundo, aunque hay algo difícilmente exportable, la generosidad y altruismo que constituyen las señas de identidad de nuestro pueblo navarro.

\section{BIBLIOGRAFÍA}

1. Memoria 2002. Comunidad Foral de Navarra. Programa de Trasplantes. Ripoll F, pg. 46.

2. Memoria 2002. Comunidad Foral de Navarra. Programa de Trasplantes. Ripoll F, pg. 58.

3. Revista Española de Trasplantes, 2005; 4:247.

4. Newsletter Transplant 2005; 10 (1): 7.

5. Newsletter Transplant 2005; 10 (1): 17.

6 . The Journal of European Transplant Coordinators Organization 2004; 7 (2): 102.

7. The Journal of European Transplant Coordinators Organization 2006; 9 (2): 80.

8. The Journal of European Transplant Ccoordinators Organization 2004; 7 (2): 116.

9. Revista Española de Trasplantes 2005; 14 (4): 248.
10. Memoria 2002. Comunidad Foral de Navarra. Programa de Trasplantes. Ripoll F, pg. 102.

11. Revista Española de Trasplantes 2005; 14 (2): 74

12. Revista Española de Trasplantes 2005; 14 (4): 249.

13. Memoria 2002. Comunidad Foral de Navarra. Programa de Trasplantes. Ripoll F, página 65.

14. Revista Española de Trasplantes 2005; 14 (4): 250

15. Revista Española de Trasplantes 2005; 14 (2): 114

16. Revista Española de Trasplantes 2005; 14 (2): 128

17. Coordination and donation of "Intra and Extra Hospital" Cadáver Donors. The Pamplona's Model. Sequence of Tasks Performed 1992-2004. $9^{\circ}$ Congreso of the WFSICCM-Awards. August 27 to 31, 2005. Buenos Aires. Argentina.

18. ECO RENAL 2006; 50: 8.

19. Revista Española de Trasplantes 2005; 14 (3): 157

20. Revista Española de Trasplantes 2005; 14 (3): 135.

21. Memoria 2002. Comunidad Foral de Navarra. Programa de Trasplantes. Ripoll F, pg. 113.

22. Revista Española de Trasplantes 2005; 14 (3): 129 .

23. Revista Española de Trasplantes 2005; 14 (3): 150.

24. Revista Española de Trasplantes 2005; 14 (3): 151.

25. Revista Española de Trasplantes 2005; 14 (3): 144

26. Memoria 2002. Comunidad Foral de Navarra. Programa de Trasplantes. Ripoll F, pg. 127. 